\title{
Precise excision of telomere-bearing transposons during Oxytricha fallax macronuclear development
}

\author{
Deborah J. Hunter, Kevin Williams, Samuel Cartinhour, ${ }^{1}$ and Glenn Herrick ${ }^{2}$ \\ Cellular, Viral, and Molecular Biology, University of Utah School of Medicine, Salt Lake City, Utah 84132 USA
}

In ciliated protozoa, development of the macronucleus from a copy of the germ line micronucleus involves elimination of a large number of sequences by DNA splicing akin to precise excision of transposons. The known examples of such internal eliminated sequences (IESs) are not repetitive. The telomere-bearing elements (TBE1s) of Oxytricha fallax are a family of transposons. We show that two particular TBE1s are also IESs. TBE1-1 and TBE1-2 disrupt a micronuclear region that codes for macronuclear DNA. A variety of tests indicates that each TBE1 and one copy of the flanking target repeat is absent from most, if not all, molecules of the macronuclear DNA, as if the TBE1s were precisely excised during macronuclear development. Three alternative explanations for the absence of TBE1-1 and TBE1-2 from the macronuclear DNA were tested. First, because two other highly homologous versions of that DNA are also found in the macronucleus, recombination between versions during or after macronuclear development could have bypassed the elements. Recombination in the regions flanking the elements was not detected. Second, micronuclear DNA blots show no evidence of a micronuclear counterpart of the macronuclear region that lacks TBE1-1. Third, TBE1-2 was demonstrated in two sexually independent cell lines. This shows that it pre-existed in the germ line, as opposed to having transposed into the micronuclear DNA subsequent to the generation of the macronucleus of the vegetative line that is usually studied. We conclude that TBE1-1 and TBE1-2, and possibly many of the other $\sim 1900$ micronucleus-limited TBE1s are excised as IESs during macronuclear development. These transposons appear to enjoy the luxury of relaxed constraints on family expansion, because they are removed from the genome before it is expressed. We discuss the possibility that all IESs are transposon-derived, that all are excised by transposition machinery, and that linear excision products are early intermediates in transposition.

[Key Words: Oxytricha fallax; ciliate transposons; TBE1s; IESs]

Received September 15, 1989; revised version accepted October 16, 1989.

Ciliated protozoa interpose genomic reorganization, including massive sequence elimination, between the inheritance of germ line information and its expression. This genomic editing takes place in the developing macronucleus at the beginning of the sexual life of the organism, immediately after mating, meiosis, and crossfertilization. While the macronucleus is developing from one mitotic product of the zygotic nucleus, another mitotic product is set aside as the new germ line micronucleus. Once equipped with a new micronucleus and a mature new macronucleus, the exconjugant grows and divides clonally, duplicating the micronucleus and macronucleus of each vegetative cell cycle. The resultant clonal cell line is called a karyonide. The mature macronucleus is often considered a somatic nucleus, because most, if not all, gene expression occurs from its genome, whereas the micronucleus is silent and serves

\footnotetext{
${ }^{1}$ Current address: Department of Molecular Biology and Microbiology, Tufts University, 136 Harrison Avenue, Boston, Massachusetts 02111 USA.

${ }^{2}$ Corresponding author.
}

primarily to carry the germ line through expansion to the next conjugation (for recent reviews of ciliate molecular biology, see Steinbrück 1986; Gall 1987; Yao 1989).

Three major classes of genomic alterations occur in the developing macronucleus: chromosome breakage with attendant telomere formation, the deletion or splicing out of interstitial sequences (for review, see Yao 1989|, and amplifications both before and after these rearrangement steps. We have studied these processes as they apply to the generation of the macronuclear chromosomes of the Oxytricha fallax 81-MAC family (Cartinhour and Herrick 1984; Herrick et al. 1985, 1987a). Figure 1A provides a visual summary of various aspects of the generation of the 81-MAC family of macronuclear chromosomes. Three slightly different versions of the 81-MAC family sequence exist in the germ line; that is, there are three very similar regions in the micronucleus of each cell line studied; their genetic relationship is not known, but-assuming micronuclear diploidy-they must represent at least two nonallelic loci. These three sequence versions are referred to as vA, vB, and vC. Previously, we showed that $\mathrm{vA}$ and $\mathrm{vB}$ are each represented 
Hunter et al.

A.
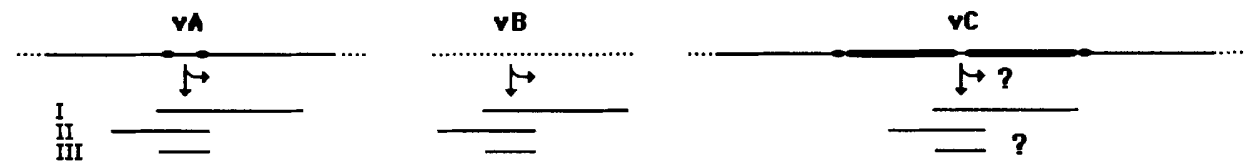

B.

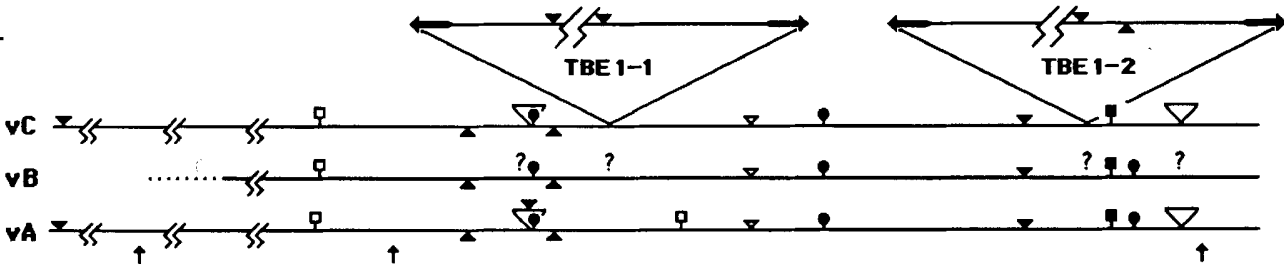

C.

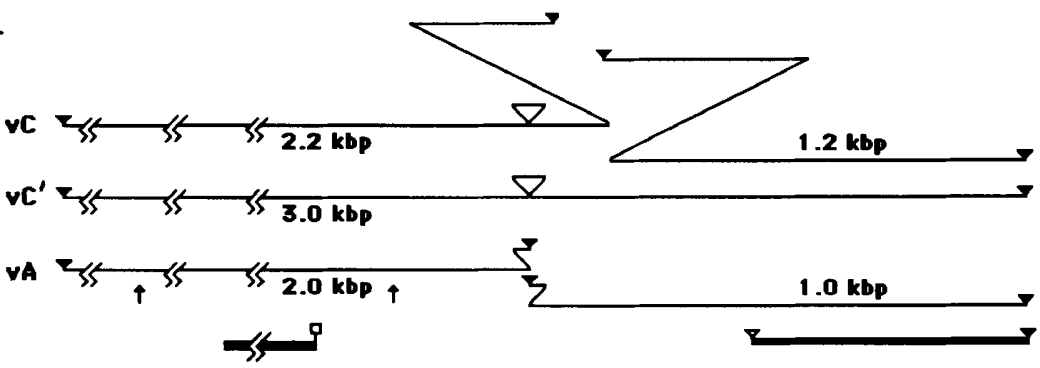

D.
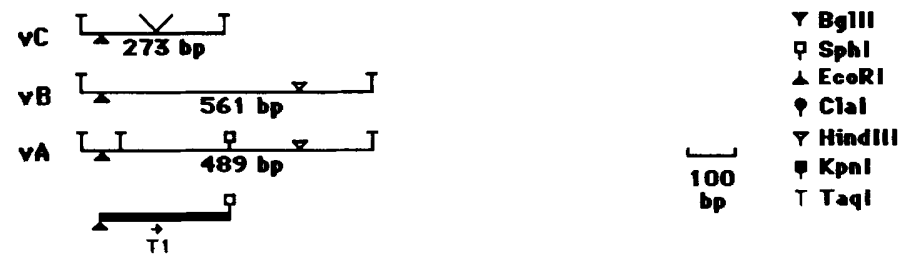

Figure 1. Maps of the 81-MAC family of sequences. $(A) \mathrm{A}$ cartoon (not to scale) representing alternative processing of each of the three versions, $\mathrm{vA}, \mathrm{vB}$, and $\mathrm{vC}$, as they are processed from their germ line or micronuclear configurations (top lines; $\mathrm{vB}$ germ line structure unknown) to their respective subfamilies of three macronuclear chromosomes. Thickened portions of the top lines represent micronucleus-limited regions (IESs and IES-like structures); the two large such regions in vC represent TBE1-1 and TBE1-2. (B) Restriction maps. Micronucleus-limited regions (IESs and IES-like structures, including TBE 1-1 and TBE 1-2) are represented as inserts above the main lines, whereas macronuclear regions are represented by the main lines. The vC macronuclear map is not derived from cloned macronuclear DNAs; it is supported by the data in this paper. The vB map is deduced solely from macronuclear DNA structures and analogy with the other versions; question marks indicate that the presence of IESs is unknown. Positions of alternative breakage and telomere addition are indicated by arrows below the vA map; the rightward two arrows define the $\sim 1.6-\mathrm{kb}$ region common to all three size classes of macronuclear chromosomes (see Herrick et al. 1987a). (C) Micronuclear DNA Bg/II fragments detected and not detected in blots shown in Fig. 6. The 3.0-kb fragment labeled vC' is not detected in Fig. 6 (see text). The probes used in that experiment are represented by the thickened bars. $(D)$ Macronuclear TaqI fragments seen in the experiments shown in Figs. 3 and 4. Probes for these experiments are represented below as the thickened bar and as the $\mathrm{Tl}$ arrow; the latter indicates the position and polarity of the $\mathrm{Tl}$ oligomer.

in the macronucleus by a set of three macronuclear chromosomes. The chromosomes are extremely short by usual standards $(\sim 4.8, \sim 2.8$, and $\sim 1.6 \mathrm{~kb})$ but are typical for macronuclear chromosomes of hypotrichous ciliates, of which $O$. fallax is a representative species. The chromosomes of a version are formed as a result of the use of alternative sites of chromosome breakage and telomere addition (Herrick et al. 1987a). The three chromosomes of a version share an identical region of $\sim 1.6 \mathrm{~kb}$ : The smallest chromosome consists exclusively of this common region (plus telomeres), whereas the larger chromosomes have an arm appended to one end or the other end of the common region. The common region bears a gene for a protein homologous to the ADP/ATP carriers of plants and metazoans (Herrick et al. 1987; G. Herrick, in prep.). In this report we show that $\mathrm{vC}$ is also alternatively processed to give rise to a set of three chromosomes, so that, in all, the 81-MAC family is represented in the macronucleus by three sets of three chromosomes each. The major finding in this study, however, concerns interstitial deletion; that is, the splicing out of internal eliminated sequences (IESs). IES excision appears to take place directly at the DNA level (Jahn et al. 1989; Tausta and Klobutcher 1989|. 
Here, we explore the possibility that IESs are transposons and transposon relics and that their excision during macronuclear development is transposase mediated. The notion that IESs are or were transposons has been suggested previously (Klobutcher et al. 1984; Herrick et al. 1985), and was based on two sets of observations. First, IESs are bounded by short direct repeats, as if created by transposition with attendant target site duplication. One of the repeats is deleted along with the IES (Klobutcher et al. 1984). In transposon terms this type of deletion is called precise excision. Second, during our studies of the 81-MAC family in $O$. fallax, we discovered a novel type of transposons, the telomere bearing elements (TBE1s). TBE1s cause target-site duplications, and all members are eliminated during macronuclear development. This suggested to us that these transposons are IESs, although elimination by other pathways was not excluded, because we had no evidence that they interrupt macronuclear-destined sequences (Herrick et al. 1985). Fresh support for the idea of transposons being IESs has come from recent work in $\mathrm{Eu}$ plotes crassus (Jahn et al. 1988; Baird et al. 1989; see Discussion).

TBEls have not been observed to transpose in the laboratory, but do appear to be transposons (Herrick et al. 1985). The elements comprise a family of repetitive micronuclear sequences with $\sim 1900$ members, consistent with their being transposons. More convincing evidence came from two sets of observations of two TBEls of $\sim 4$ $\mathrm{kb}$, which were named TBE1-1 and TBE1-2 and which were found interrupting the micronuclear sequences of the 81-MAC family. First, although these elements interrupt $\mathrm{vC}$ micronuclear DNA, they are not found in vA micronuclear DNA (Fig. 1A, B); we have no cloned vB micronuclear DNA, but analysis of polymerase chain reaction (PCR) products indicates (see Results) that TBE 1-2 is also absent from vB as well as from vA. vB and $\mathrm{vC}$ are more similar to each other than to vA /see Results), which suggests that vA diverged from a precursor of $\mathrm{vB}+\mathrm{vC}$, which later gave rise to $\mathrm{vB}$ and $\mathrm{vC}$. The clear implication is that the elements interrupt $\mathrm{vC}$ as a result of transposition, as opposed to pre-existing elements having been independently lost from vA and vB. Second, the elements are bounded by 3-bp direct repeats, interpreted to be typical target duplications, and have 77/78-bp inverted terminal repeats. The distal $17 \mathrm{bp}$ of the inverted terminal repeats consists of $\mathrm{dC}_{4} \mathrm{~A}_{4}$ repeats. These unusual sequences are normally found at the telomeres of hypotrich chromosomes, which suggested to us that transposition may involve a linear intermediate (Herrick et al. 1985). Cherry and Blackburn (1985) characterized a Tetrahymena repetitive sequence element called Tel-1 and were led to a similar proposal.

We extended the analysis of TBE1-1 and TBE1-2 and their flanking $\mathrm{vC}$ sequences to test the hypothesis that the two TBEl elements are IESs. We found that their flanking $\mathrm{vC}$ sequences are present in macronuclear DNA at normal copy number, whereas each TBE 1 - and one of its flanking target repeats-is absent, suggesting that they were precisely excised. Evidence was obtained against three alternative explanations for this absence, leading us to conclude that TBE1-1 and TBE1-2 are IESs.

\section{Results}

Tests for the presence in macronuclear DNA of the $\mathrm{VC}$ micronuclear sequences that flank TBE-1 and TBE1-2

We lacked evidence that $\mathrm{vC}$ sequences are in the macronucleus, because no cloned vC macronuclear DNAs have been obtained. Versions A and B are highly homologous to $\mathrm{vC}$ and are present in the macronucleus. Therefore, we had to design specific assays for macronuclear $\mathrm{vC}$ DNA that could distinguish $\mathrm{vC}$ from $\mathrm{vA}$ and $\mathrm{vB}$. The extent of similarity between versions is immediately evident in their restriction maps (Fig. 1B).

Representatives of each version have been cloned from DNA of the karyonidal cell line 3.5 (micronuclear vC, macronuclear $\mathrm{vB}$, and both micronuclear and macronuclear vA), and major portions of each clone have been sequenced previously (Herrick et al. 1985, 1987a, 1987b; see Figure $5 \mathrm{~A}$, below). The versions differ primarily by scattered single nucleotide mismatches. A detailed comparison of one region is shown in Figure 5A (below). In all, a large number of differences have been identified. Of $1660 \mathrm{bp}$ compared between all three versions, vA differs from $\mathrm{vB}$ and $\mathrm{vC}$ by $4.3 \%$ and $4.9 \%$ (71 and $81 \mathrm{bp}$ ), respectively, whereas $\mathrm{vB}$ and $\mathrm{vC}$ differ only by $3.2 \%(53$ bp). Based on these differences, three distinct assays were developed to detect macronuclear vC DNA: (1) vCspecific oligomer hybridizations; (2) detection of $\mathrm{vC}$-specific restriction fragments, and (3) DNA sequencing.

$v C$-specific oligomer hybridizations Because macronuclear chromosomes are so small, the chromosomes are well resolved by standard agarose gel electrophoresis, which was used to fractionate undigested macronuclear or whole-cell DNAs. The blot of a gel was hybridized with the 24-nucleotide oligomer oCl-88, which was designed to specifically detect $\mathrm{vC}$ sequences (see Materials and methods; Fig. 5A, below). As expected, it hybridized to cloned $\mathrm{vC}$ (micronuclear) DNA and not to cloned vA or $\mathrm{vB}$ control DNAs (Fig. 2, lane 1). The probe hybridized to macronuclear chromosomes in DNA of karyonidal cell lines 3.5, 9D1, and 9D2 (Fig. 2, lanes 2-4). Two aspects of these results are noted.

First, although not relevant to the issue at hand, three different sizes of chromosomes are detected (the fourth band in 3.5 DNA is rDNA; see legend to Fig. 2). Because there only appears to be only a single vC-like micronuclear region (Herrick et al. 1985; see also below), this result indicates that $\mathrm{vC}$ sequences are alternatively processed, as vA and vB already have been shown to be processed (Herrick et al. 1987a). As with the other two versions, the copy number of the smallest chromosome is considerably higher than that of the two larger ones. This blot was rehybridized with a vA-specific oligomer; the $\mathrm{vA}$ and $\mathrm{vC}$ chromosomes had indistinguishable mobilities (not shown). The vA chromosomes and, hence, the $\mathrm{vC}$ chromosomes, are $\sim 4.8, \sim 2.8$, and $\sim 1.6 \mathrm{~kb}$ linear 


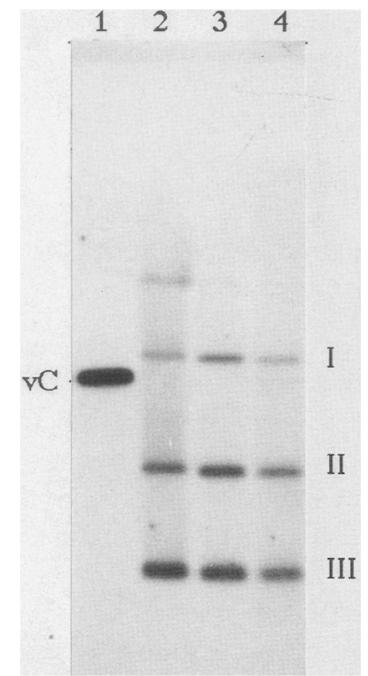

Figure 2. Version $\mathrm{C}$ macronuclear chromosomes in three karyonides. Autoradiograph of a Southern blot of a $0.7 \%$ agarose electrophoresis gel hybridized at $40^{\circ} \mathrm{C}$ with $5^{\prime}-32 \mathrm{P}$-labeled oCl-88, as described in Materials and methods, with a $44^{\circ} \mathrm{C}$ 3 -min final wash. Lane 1 carried an equimolar mixture of cloned DNA fragments representing the three versions mixed with $10 \mu \mathrm{g}$ of EcoRI-digested calf thymus DNA; the mixture was composed at a high concentration, and its composition confirmed by ethidium bromide staining of an electrophoresis gel (not shown): (vA) $1.2 \mathrm{ng}$ of a $6.0-\mathrm{kb}$ linear form of pMA83s (Herrick et al. 1987a); (vB) $0.34 \mathrm{ng}$ of the 1.7-kb PstI fragment of pMA83 (Cartinhour and Herrick 1984); (vC) $0.91 \mathrm{ng}$ of the 4.5$\mathrm{kb}$ linear form of the plasmid HG-Ø8 (pEMBL 8 + vector; Dente et al. 1983), a subclone from the micronuclear $\lambda$ clone L47-08. Lanes 2-4 carried $10 \mu \mathrm{g}$ each of 3.5 macronuclear DNA, 9D1 whole-cell DNA, and 9D2 whole-cell DNA, heated to $55^{\circ} \mathrm{C}$ prior to loading. In addition to bands of the three 81-MAC chromosomes, MAC I, II, and III, another band is seen in the 3.5 DNA; it comigrates with the massive band of rDNA chromosomes and represents nonspecific hybridization (for full discussion, see Herrick et al. 1987b).

chromosomes (Herrick et al. 1987a). Three different karyonidal cell lines represent independent sexual progeny and independent episodes of macronuclear development. $\mathrm{vC}$ is found in all three cell lines (Fig. 2, lanes 2-4), as are vA and vB (Herrick et al. 1987b; D. Hunter, unpubl.). In summary, the 81-MAC family is reproducibly represented in three independent karyonidal cell lines by a set of nine distinct macronuclear chromosomes-three from each of three versions.

More importantly, these results provide evidence that micronuclear $\mathrm{vC}$ sequences lying between TBEl-1 and TBE1-2 are present in macronuclear DNA. Another vCspecific oligomer, the 16-mer oC-RCR, which hybridizes to sequences to the right of TBE1-2 (see Fig. 5A, below), also specifically hybridized to the three sizes of macronuclear chromosomes in a similar experiment (not shown). Together, the 24-mer oCl-88 and the 16-mer oC-RCR hybridize to 40 nucleotides, of which 17 are diagnostic for $\mathrm{vC}$.

$v C$-specific restriction fragments The map in Figure ID shows that $\mathrm{vC}$ macronuclear DNA should yield a diagnostic 273-bp TaqI fragment (if TBE1-1 is absent). TaqI-digested macronuclear DNA from karyonidal cell line 3.5 was subjected to electrophoresis, blotted, and hybridized with an appropriate restriction fragment (for description of the probe, see Fig. 1D). The autoradiograph (Fig. 3) shows the predicted band, migrating just slightly slower than a 267-bp size standard included in the gel sample (the $\sim 273$-bp fragment has also been observed in digests of DNAs from karyonides 9D1 and 9D2; not shown). vA and vB TaqI fragments were also seen, comigrating with fragments from authentic cloned DNAs. The copy numbers of the three versions are roughly equal (these TaqI fragments are derived from all three macronuclear chromosome size classes, being cut from within the common region). In any case, the demonstration of the 273-bp TaqI fragment provides further evidence of the presence of $\mathrm{vC}$ sequences in macronuclear DNA. In addition, the vC-specific absence of a $\mathrm{ClaI}$ site was demonstrated in macronuclear DNA /see below).

Thus, oligonucleotide hybridizations and restriction

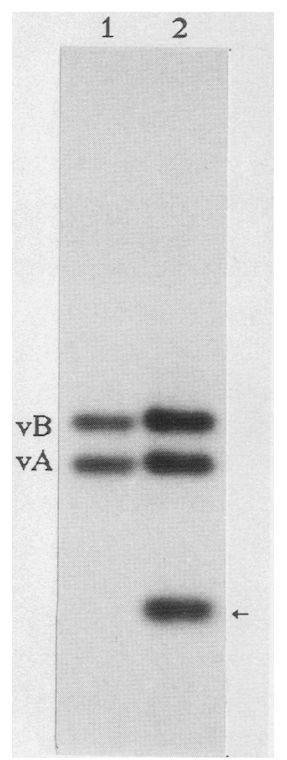

Figure 3. A vC-specific TaqI fragment from macronuclear DNA. Autoradiograph of a Southern blot of a $2.0 \%$ agarose electrophoresis gel hybridized and washed as described in Materials and methods; hybridization and stringent wash temperatures were 40 and $44^{\circ} \mathrm{C}$. (See Fig. 1D for TaqI maps.) Lane 1 carried an equimolar mixture of TaqI-digested vA and vB plasmid DNAs mixed with $10 \mu \mathrm{g}$ of sonicated calf thymus DNA: (vA) $1.2 \mathrm{ng}$ of digested pMA83s (Herrick et al. 1987a); (vB) 0.93 ng of digested $83 \mathrm{~B}-2$, a plasmid carrying the $P$ stI insert of pMA83 (Cartinhour and Herrick 1984) in the Bluescript $\mathrm{KS}+$ vector (Stratagene). Lane 2 carried $10 \mu \mathrm{g}$ of digested 3.5 macronuclear DNA and 5 ng of HaeIII-digested pBR322. The probe (see Fig. 1D) was the gel-purified EcoRI/SphI fragment from the pEMBL8 + plasmid 21-3-1, which carries the EcoRI/HindIII fragment of the micronuclear vA $\lambda$ clone L47-21 (Herrick et al. 1985). The fragment was labeled with the random-primer reaction. The arrow to the right of lane 2 indicates the position of the 267-bp HaeIII pBR322 fragment in lane 2 visualized by appropriate rehybridization. 
analyses have demonstrated $\mathrm{vC}$ features in macronuclear DNA. In a later section we present the results of sequencing macronuclear DNA produced by the polymerase chain reaction (PCR). At each of 44 positions examined, it showed $\mathrm{vC}$-specific nucleotides diagnostic of $\mathrm{vC}$. In all, $54 \mathrm{vC}$-specific nucleotides have been found in macronuclear DNA. In summary, a variety of data leads to the conclusion that $\mathrm{vC}$ sequences and, hence, the micronuclear sequences that flank TBE1-1 and TBE1-2 are represented in macronuclear DNA.

\section{Absence of TBE1-1 and TBE1-2 in $v C$ macronuclear DNA}

In this section we present results arguing that each TBE1 - and one of its flanking target repeats-is absent from most, if not all, $\mathrm{vC}$ macronuclear DNA molecules, showing that both TBE1-1 and TBE1-2 were, by definition, precisely excised. Information about TBE 1-1 comes from two analyses of the vC TaqI fragment discussed above, whereas information about TBE1-2 comes from the DNA sequencing just mentioned.

The size of the vC TaqI fragment is quite close to 273 bp (Fig. 3), which is consistent with the absence of TBE1-1 in vC macronuclear DNA (Fig. ID). To investigate more closely, we designed the 21-nucleotide oligomer Tl (see Fig. 1D). Its sequence spans the junction that would be created by the removal of TBE1-1 and one AAT target repeat from the micronuclear $\mathrm{vC}$ sequence. The $\mathrm{Tl}$ sequence is the same as the known sequence of macronuclear $\mathrm{vA}$ and $\mathrm{vB}$ at that position. TaqI-digested macronuclear DNA was loaded in four lanes of an agarose gel, subjected to electrophoresis, blotted, and probed with this oligomer. The blot was washed non-

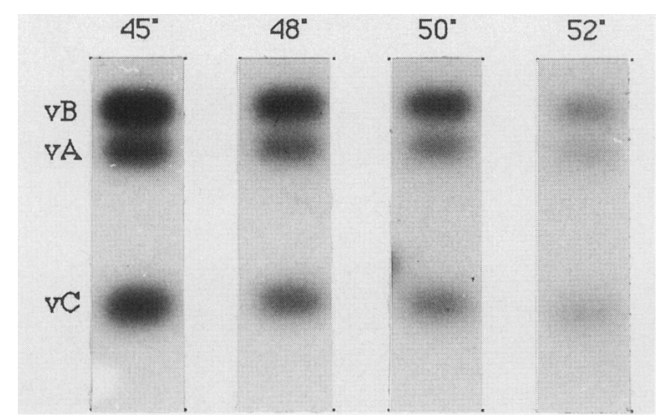

Figure 4. Thermal stability of the TBE1-1-less vC macronuclear DNA region and the T1 oligomer. Autoradiograph of strips of a Southern blot of a $2.0 \%$ agarose electrophoresis gel hybridized at $35^{\circ} \mathrm{C}$ with $5^{\prime}-32 \mathrm{P}$-labeled T1 oligomer (Fig. 1D) as described in Materials and methods. Strips bearing lanes carrying $3.3 \mu \mathrm{g}$ of TaqI-digested 3.5 macronuclear DNA were washed at $45,48,50$, and $52^{\circ} \mathrm{C}$ for $3 \mathrm{~min}$. The washed strips were then reassembled side by side, and an autoradiograph was produced and photographed. Portions of a single photograph of the autoradiograph are shown; no bands were omitted. The bands seen correspond to the three bands seen in Fig. 3. The vA band is slightly underrepresented (cf. to Fig. 3); this is likely attributable to slightly incomplete TaqI digestion in the present case. (See Fig. 1D for TaqI maps). stringently and cut into four strips; the strips were washed at $45,48,50$, and $52^{\circ} \mathrm{C}$ (Fig. 4). The melt-off temperature profile of the oligomer from the $\sim 273$-bp band is indistinguishable from those from the $\mathrm{vA}$ and $\mathrm{vB}$ bands; that is, the relative intensity of the $\sim 273$-bp vC band, compared with the $\mathrm{vA}$ and $\mathrm{vB}$ bands, is apparently constant from strip to strip. This suggests that those oligomer-vC hybrids that formed are as strong as the perfect duplexes formed with $\mathrm{vA}$ and $\mathrm{vB}$ and suggests that TBE1-1 was precisely excised. It is difficult to quantitatively evaluate the extent of match from these melt-off data, but our experiences with mismatches strongly suggest to us that only perfect $\mathrm{vC}-\mathrm{Tl}$ hybrids are seen in this experiment. It is possible that a population of $\mathrm{vC}$ macronuclear DNAs exist from which TBE1-1 was sufficiently imprecisely excised (e.g., because of variability in excision) that the oligomer might not even hybridize to some of those molecules and would not be detected in this experiment; however, we see no evidence for such molecules. A restriction fragment probe (see Fig. 1D) was hybridized to one of the blot strips; it should detect such imprecisely derived vC TaqI fragments, because it is large and its hybridization should be insensitive to even major mismatches at the junction. The relative intensity of the $\mathrm{vC}$ band versus $\mathrm{vA}$ and $\mathrm{vB}$ bands (not shown) was indistinguishable from that seen with the $\mathrm{T} 1$ oligomer probe. It seems likely that most of the $\mathrm{vC}$ macronuclear DNA molecules in the preparation have the sequence that would be generated by precise excision of TBE1-1.

The most direct way to examine the issue is to isolate $\mathrm{vC}$ macronuclear DNA and determine the nucleotide sequence at the site of excision. We set out to do this for the case of TBE1-2. To obtain putative $\mathrm{vC}$ macronuclear DNA, we took advantage of the $\mathrm{vC}$-specific absence of a ClaI site (right end of map, Figs. $1 \mathrm{~B}$ and $5 \mathrm{~A}$; the vC sequence is ATCaAT versus ATCGAT in vA and vB) and used the PCR to produce ClaI-resistant DNA. Primers PCR-L1 and PCR-R1 (Fig. 5A) span this position (568 bp, including primers), and ClaI-cut molecules will not support the PCR reaction. Because the $\mathrm{vA}$ and $\mathrm{vB}$ ClaI site is fully protected from ClaI digestion in DNA isolated from macronuclei, probably by macronuclear dA methylation (Cartinhour and Herrick 1984), a first round of PCR (30 thermal cycles) was run on undigested macronuclear DNA. The PCR product (now unmethylated) was then cut with ClaI, and the resistant band was gel purified. That material was further amplified by PCR and ClaI-resistant material purified. Again, ClaI-resistant material was amplified, and ClaI-resistant material was isolated.

This triply ClaI-challenged material was subjected directly to sequencing with a variety of primers (Fig. 5A). A sequence of 467 nucleotides was determined. The sequencing confirms that the DNA sequenced is vC DNA in a variety of ways; that is, its sequence matches micronuclear $\mathrm{vC}$ perfectly: only $\mathrm{vC}$-specific nucleotides were found -44 in all. These results are represented in Figure 5A. Figure 5B shows a portion of an autoradiograph generated using $\mathrm{PE}-\mathrm{B}$ as a sequencing primer, and 


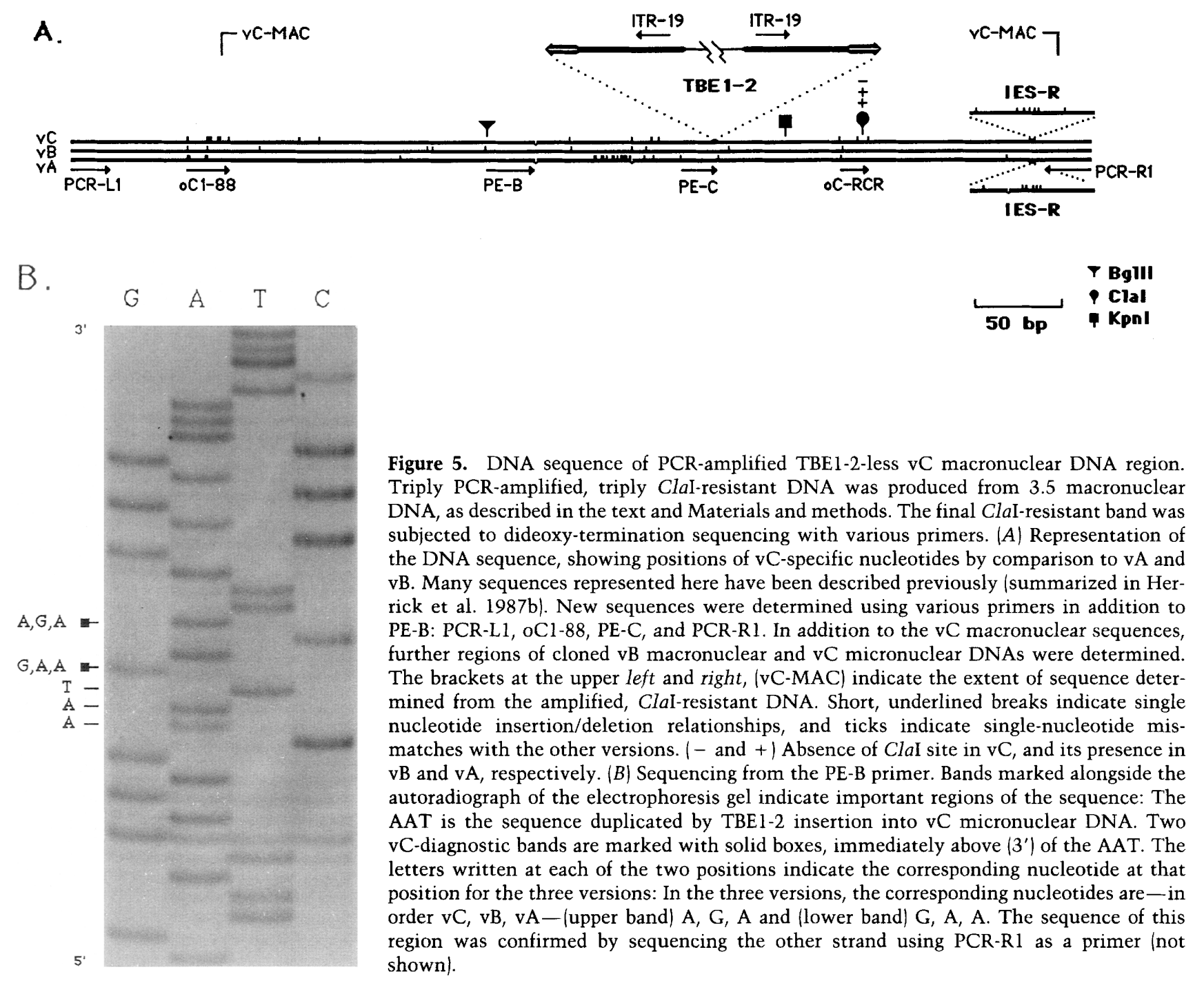

two bands representing $\mathrm{vC}$-specific nucleotides are marked. Second, the distance from the PE-B primer to the area read is different in each of the three versions, because of single nucleotide insertion/deletion differences near the primer (Fig. 5A). The fact that a sequence can be read at all shows that only one version-clearly, $\mathrm{vC}$ - is represented; indeed, in a side-by-side run, sequencing ladders from PE-B for cloned vA and vB DNAs were displaced from each other in the predicted way (not shown). Finally, the vC-specific oligomer oC1-88 functioned as a good primer for sequencing of the amplified DNA but not for sequencing of a vB control DNA /not shown|, confirming that this DNA is vC DNA.

Although DNA from purified macronuclei was used for the PCR amplification, the VC DNA obtained may have been derived from contaminating micronuclear DNA. vC micronuclear DNA carries a 70-bp IES-like element very close to the right end of the amplified region
(Herrick et al. 1987b; Fig. 5A). Sequencing of the vC PCR DNA showed clearly that this short element and one flanking direct repeat are absent (not shown). This result not only confirms that the DNA is macronuclear but it also demonstrates that the 70 -bp element is a true IES with typical IES sequence properties.

More importantly, however, the sequencing results (Fig. 5B) show that TBE1-2 is absent from vC macronuclear chromosomes. Not only is TBE 1-2 absent from the nucleotide (the element begins 5'-CAAAACCCC- and ends -GGGGTTTTG-3'; Herrick et al. 1985), but only one copy of AAT target sequence duplicated by TBE1-2 insertion into $\mathrm{vC}$ micronuclear DNA is present. Note that unlike DNA cloning, PCR amplifies a large number of molecules, showing that TBE1-2 and one AAT are absent from most, if not all, of the $\mathrm{vC}$ macronuclear chromosomes in the preparation examined: Variability in precision of excision would have caused ambiguity in 
the sequencing ladder distal $\left(3^{\prime}\right)$ to the AAT, which was not observed.

Possible reasons for the absence of TBE1-1 and TBE1-2 from $v C$ macronuclear DNA

One explanation for the absence of the TBEls from vC macronuclear DNA is that they are precisely excised as IESs during macronuclear development. Three alternative explanations have been considered. First, macronuclear recombination has been demonstrated recently between macronuclear rDNAs in Tetrahymena (L $\varnothing$ vlie et al. 1988; Yu et al. 1988; Sweeney and Yao 1989; Yaeger et al. 1989). It was conceivable that TBE1-1 and TBE1-2 are absent from macronuclear DNA as a result of fortuitous crossovers between $\mathrm{vC}$ and either vA and vB sequences, during macronuclear development or during subsequent propagation. The various results above seem to eliminate this possibility. We see no evidence for recombination on either side of either TBE1 element. As just shown, essentially all the molecules produced by the PCR reaction are purely vC over the 467-bp tested. Of the $44 \mathrm{vC}$-specific nucleotides detected, 36 lie to the left of the TBE1-2 site, and 8 lie to the right. The TaqI blots shown earlier (Figs. 3 and 4) also allow us to examine large numbers of macronuclear DNA molecules, and we see no evidence for recombination between the polymorphic TaqI markers that flank the TBE1-1 target. To our knowledge, these results represent the only information bearing on macronuclear recombination in hypotrichous ciliates.

A second potential explanation for the absence of the TBEls in vC macronuclear DNA is that it may have been generated from a vC-like germ line region lacking TBE1-1 and TBE1-2, although no clones of such DNA were obtained from $\lambda$ L47 micronuclear DNA clone libraries (Herrick et al. 1985). To look for such a DNA, BglII-digested DNA from purified micronuclei of karyonide 3.5 was subjected to electrophoresis, blotted, and probed with two different probes. The maps in Figure 1C define the probes and show the rationale for the test. The results are shown in Figure 6. vC micronuclear DNA has a 72-bp IES-like element just left of TBE1-1. vA has a homologous IES at the corresponding position. The vA IES has a BglII site, but the vC sequence does not, the site differing by two nucleotides (AGATCT versus TAATCT; Herrick et al. 1987b). The vC fragments that terminate in the two ends of TBE1-1 cannot be seen in the micronuclear DNA digests because of fragments that were generated from contaminating macronuclear DNA and that fortuitously comigrate with the vC micronuclear DNA fragments. Nonetheless, the vA micronuclear DNA fragments to either side of that $B g I I I$ site can be seen clearly in areas free of macronuclear DNA contamination $(2.0 \mathrm{~kb}$ in Fig. 6A, lane $3 ; 1.0$ $\mathrm{kb}$ in Fig. 6B, lane 2). If vC-like micronuclear DNA, that is, with a BgIII-less IES, lacking TBE1-1 existed, it would generate a $3.0-\mathrm{kb}$ fragment that would hybridize with either probe $\left(\mathrm{vC}^{\prime}\right.$ in Fig. $\left.1 \mathrm{C}\right)$. This $3.0-\mathrm{kb}$ fragment could

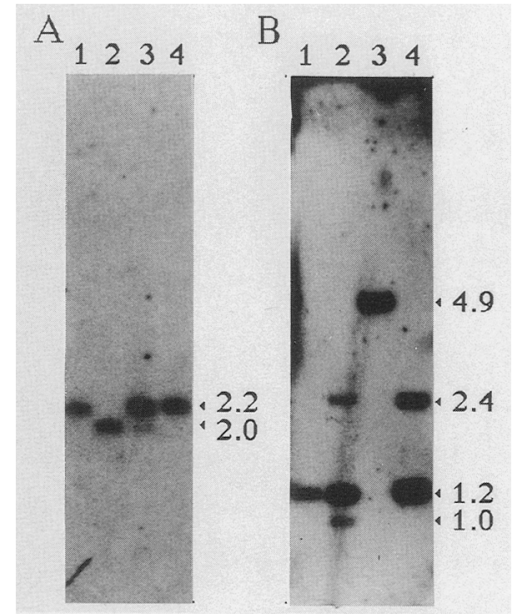

Figure 6. Southern blot tests for micronuclear vC-like DNA lacking TBE1-1. Autoradiographs of Southern blots of $0.7 \%$ agarose electrophoresis gels. Samples contained complete BglII digests of $2.0 \mu \mathrm{g}$ of 3.5 micronuclear DNA, $0.2 \mu \mathrm{g}$ of $3.5 \mathrm{ma}-$ cronuclear DNA, or $100 \mathrm{pg}$ of phage DNA. Blots were probed with nick-translated restriction fragments purified from gels. Approximate sizes of fragments are indicated. (See Fig. 1C for maps and positions of probes; see Fig. $1 \mathrm{~B}$ to discern the identities of the macronuclear fragments). (A) The probe was the $\sim 0.5$-kb PstI-SphI fragment from a pMA82d (Cartinhour and Herrick 1984) digest. (Lane 1), L47-26 (micronuclear vC clone) DNA digest; (lane 2), L47-21 (micronuclear vA clone) DNA digest; (lane 3) micronuclear DNA digest; (lane 4) macronuclear DNA digest. $(B)$ The probe was the HindIII-BgllI fragment of pMA83 (Cartinhour and Herrick 1984). (Lane 1) L47-08 (vC) DNA digest; (lane 2) micronuclear DNA digest; (lane 3) L47-04 DNA digest (this phage proved to be artifactual, and this lane is included to prevent cutting of the photograph); (lane 4) macronuclear DNA digest.

have been seen but was not detected on either blot (Fig. $6 \mathrm{~A}$, lane 3 ; Fig. 6B, lane 2). It cannot be ruled out completely, however, that such a germ line DNA did exist but was subsequently lost from the micronucleus during clonal expansion prior to harvest of the micronuclei analyzed.

Finally, we considered the possibility that TBE1-1 and TBE1-2 transposed into the micronuclear vC region subsequent to the time that the macronucleus developed in the exconjugant, which gave rise to the karyonide 3.5, from which all our micronuclear DNAs have been isolated. That is, at the time vC DNA was processed, TBE1-1 and TBE1-2 were absent from vC, explaining their absence from $v C$ macronuclear DNA. To test this, evidence for the prior existence of TBE 1-2 in VC DNA was sought by PCR analysis of whole-cell DNA from the independent karyonidal cell line 9D1. Figure 5A shows the positions of the primers used. The ITR-19 oligomer primes synthesis from within each inverted terminal repeat of TBE1-2, outward toward either PCR-L1 or PCR-R1, and products of 427 and 282-bp should be produced if TBE 1-2 exists in vC micronuclear DNA. Figure 7 shows that products of approximately the expected size were generated (lanes 3,5 , and 8 ). DNA sequencing (not shown) of the leftward product using ITR-19 as the 


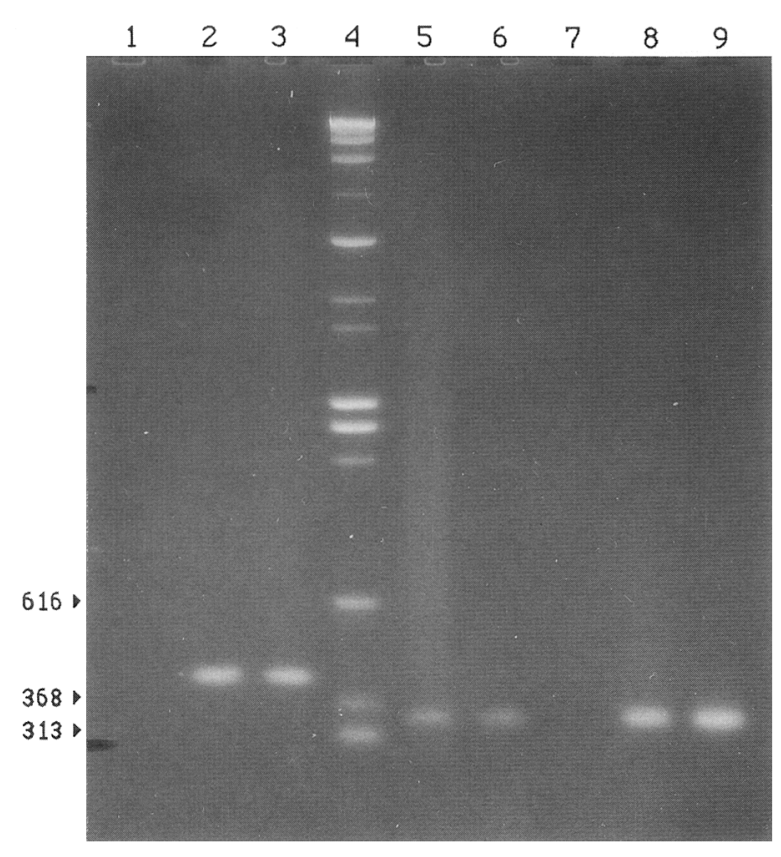

Figure 7. PCR tests for existence of TBE1-2 in karyonide 9D1. Ethidium-bromide stained $2.0 \%$ agarose electrophoresis gel of products of PCR reactions primed by PCR-L1 and ITR-19 (lanes $1-3$ ) or by ITR-19 and PCR-R1 (lanes $5-9$ ); $4 \mu \mathrm{l}$ of each $50 \mu \mathrm{l}$ reaction was loaded. PCR-LI-ITR-19 templates: (Lane 1) No template DNA; (lane 2) 2.35 pg of L47-22 DNA (3.5 micronuclear vC clone; Herrick et al. 1985), which bears the right end of TBE 1-1, the center of the common region, all of TBE 1-2, and extends beyond the end of the common region (see Fig. 1B); (lane 3) $2.6 \mu \mathrm{g}$ of 9Dl whole-cell DNA; PCR hybridization temperature was $50^{\circ}$. Lane 4 carries linear size standards, including TaqI pBR322 fragments / the sizes of smallest are indicated at the left). ITR-19-PCR-R1 templates: (Lane 5) $2.6 \mu \mathrm{g}$ 9D1 whole-cell DNA; (lane 6) 2.35 pg L47-22 DNA; (lane 7) no DNA; (lane 8) second round of PCR on $5 \mu \mathrm{l}$ of 9D1 whole-cell DNA reaction; (lane 9) second round of PCR on $5 \mu$ l of L47-22 DNA reaction; PCR hybridization temperatures were $50^{\circ} \mathrm{C}$ for the first rounds and $37^{\circ} \mathrm{C}$ for the second rounds. All PCR reactions were for 30 cycles. Contamination considerations: Notemplate control reactions gave no detectable products (lanes 1 and 7). We have isolated no plasmid clones that would support either PCR reaction, but several different $\lambda$ L47 micronuclear DNA clones, such as L47-22, have been isolated that would (Herrick et al. 1985). A PCR reaction diagnostic of the L47 left arm worked well with appropriate amounts of wild-type $\lambda$ and L47 DNAs but gave no product with 9D1 whole-cell DNA (not shown). On this basis, we feel that the PCR products shown here result from the amplification of micronuclear DNAs from 9D1 cells.

primer clearly demonstrated that the TBE1 is inserted at exactly the same nucleotide as in 3.5 DNA. It is possible that TBE1-2 also interrupts micronuclear vB DNA; however, the sequencing showed purely vC-diagnostic nucleotides (23) in the flanking sequence (not shown). Also, the rightward product was challenged with ClaI, but no cutting was observed (not shown). Karyonidal cell line 9D1, unlike 3.5, grows well only septically, so the possibility of contaminating cloned DNAs is quite real.
However, control experiments described in the legend to Figure 7 provide no evidence for contamination. To return to the main issue, in principle, it is possible that TBE1-2 was transposed independently into $\mathrm{vC}$ micronuclear sequences at exactly the same position twice, once in each of the two independent karyonidal cell lines. We consider this possibility extremely improbable and instead, conclude that TBE1-2 pre-existed-possibly for a very long time-in the O. fallax germ line and was therefore present in $\mathrm{vC}$ at the time that $\mathrm{vC}$ macronuclear DNAs were generated.

\section{Discussion}

\section{Are TBE1s internal eliminated sequences?}

Our results establish the following points. The sequences flanking TBE1-1 and TBE1-2 are present in macronuclear DNA, but TBE1-1 and TBE1-2 are absent. TBE 1-2 already interrupted the germ line vC sequence at the time that vC macronuclear DNA was generated. Presumably TBE1-1 pre-existed as well. It is unlikely that the $\mathrm{vC}$ macronuclear DNAs were generated from a germ line DNA lacking TBE1-1. Therefore, we would like to conclude that TBE1-1 and TBE1-2 are precisely excised during macronuclear development, that is, they are internal eliminated sequences (IESs). Other studies strengthen this conclusion. A study of another family of O. fallax macronuclear sequences, the pMA-H1 family, shows that an $\sim 4-\mathrm{kb}$ member of a micronucleus-limited repetitive family interrupts all micronuclear versions of the family. This suggests that the repetitive element, which does not cross-hybridize with TBE1-2, is an IES (G. Herrick, unpubl.). A family of micronucleus-limited repetitive elements was described in E. crassus and members were shown to interrupt what may be macronucleus-destined sequences (Jahn et al. 1988). Quite recently, Baird et al. (1989) reported what is most easily interpreted as the two ends of one member of this family, dubbed the Tecl elements, interrupting a proved macronucleus-destined sequence, and have firmly established that the interruption must be removed during macronuclear development. The interruption has typical transposon-like structural features: It has inverted terminal repeats flanked by short direct repeats, one of which is macronucleus destined. Given our present results and these other examples, we feel confident concluding that TBE 1-1 and TBE1-2 are IESs. All $\sim 1900$ TBE1 elements are micronucleus-limited (Herrick et al. 19851, and many of them, in addition to TBE1-1 and TBE1-2 may be IESs.

\section{Precise and imprecise, variant and invariant excision}

Our data show that TBE1s can be precisely excised. The sequencing data (Fig. 5B) firmly establish this for TBE1-2 (and the short rightward vC IES); for TBE1-1, the size of the vC TaqI fragment (Fig. 3) and the T1 melt-off data (Fig. 4) are difficult to interpret otherwise. Furthermore, essentially all of the macronuclear chromosomes in our preparations represent the products of precise excision 
events. This, however, does not necessarily mean that all TBE1-1 and TBE1-2 excisions are precise. Some IESs are known not to be excised exactly the same way in each event (Austerberry and Yao 1987; Austerberry et al. 1989|; microheterogeneity results, even within a single developing macronucleus (rearrangements occur generally only after a few rounds of amplification). We have not analyzed the immediate products of macronuclear development but, instead, have analyzed macronuclear DNAs from cells derived from exconjugants by many generations of vegetative growth. In the intervening period, the relative mix of various types of excision products may have changed. For instance, TBE1-1 interrupts a protein-coding region. If imprecise excisions occurred, many would have left the gene inoperative, and selection may have weeded out cells with a significant fraction of their chromosomes imprecisely constructed at that site. Because macronuclear genome composition is uniquely flexible, as a result of the phenomenon of macronuclear assortment, evolution of pure macronuclear genotypes from initially heterogeneous ones is to be expected, especially if a selection exists (Baird and Klobutcher 1988; for review, see Brunk 1986; Bruns 1986). Thus, our data strongly demonstrate that precise excision of TBE1-1 and TBE1-2 does occur in macronuclear development, but they allow the possibility that excision is variable, sometimes being imprecise, but at least sometimes precise.

\section{Transposition of IESs}

Special advantages for transposons that are IESs We have concluded that TBEls are both transposable and precisely excisable. In other words, macronuclear development leads to essentially complete somatic reversion of genomic disruptions caused by transposition. We view TBE1s to be special kinds of transposons that have created their own niche (for previous discussions, see Herrick et al 1985; Ribas-Aparicio et al. 1987; Jahn et al. 1988). Unlike conventional transposons, they can edit themselves (and their degenerate cousins; see below) out of the working genome. Therefore, they enjoy a special license to create new copies of themselves without major regard to location in the host genome (sequences necessary for micronuclear functions excluded). Recently, the notion of mobile introns has come to the fore, although actual interlocus intron transposition has yet to be observed experimentally (for review, see Lambowitz 1989; Scazzocchio 1989). Mobile introns would enjoy the same freedom, because, like transposable IESs, they are quantitatively and precisely removed before the final stages of gene expression.

Is IES excision mediated here by transposase? To the extent that IES excision is essential for production of a functional macronucleus, there is selective pressure to maintain the integrity of the IESs as substrates for the excision machinery, as well as the integrity of the excision machinery itself. The host may encode the excision machinery; however, the same selective pressure exists for the parasites, that is, for the TBEls and any other transposons that are also IESs, because their fate is firmly tethered to that of the host. As argued previously (Herrick et al. 1985; Ribas-Aparicio et al. 1987), all IESs may be transposable elements, or remnants thereof, which retain the cis-acting features necessary for excision during macronuclear development. By this view, autonomous elements (TBEls and other types) would maintain the genes for machinery responsible for all IES removal.

If excised TBEls are early intermediates in transposition (see below), then, by the commonly used functional definition, we would refer to its excision machinery as transposase. It might be argued that TBEl excision must not be mediated by transposase, because excision events mediated by transposases of well-characterized eukaryotic transposons often are imprecise, whereas TBE1 excisions could be predominantly precise. However, we have just noted that the actual frequency of precise TBE1 excision is not known, and some level of imprecise excision is fully possible. Furthermore, a wide range of fidelity of excision by transposases is seen in different systems. Actually, what is normally observed is only the fidelity of joining the sequences from which the transposon has been excised. Presumably the excised transposon is liberated intact and unaltered, especially in conservative transposition systems, where the excised element will be reinserted elsewhere. Tn 10 is thought to leave behind a chromatid break that is not repaired at all, surely the lowest fidelity of joining (Kleckner 1989). The best characterized plant transposons, the Drosophila P element and the Caenorhabditis elegans Tcl element, routinely leave behind imprecise joints, although the $\mathrm{P}$ element transposase does seem to participate in some level of precise excision/joining events and Tc1 joining fidelity may be site-dependent (for reviews, see Coen et al. 1989; Engels 1989; Fedoroff 1989; Moerman and Waterston 1989). In these systems, excision is a rare event, and there may not be sufficient selective pressure to evolve a high-fidelity joining mechanism. In contrast, excision is not a rare event in ciliates, which routinely depend on the fidelity of excision to make a functional macronucleus, and one might expect the transposase to have evolved to give quite a high frequency of precise excisions.

TBE1 excision in the developing macronucleus as the first step in transposition TBE 1 transposition may occur fully within the micronucleus. Other genetic activities transpire there, namely DNA repair (Ammermann 1988) and, obviously, DNA replication; however, the micronucleus is otherwise remarkably silent. Transcription appears to be fully suppressed, whereas the macronucleus is fully active in the same cytoplasm. IES excision, as well as chromosome breakage, also must be suppressed, not only in the vegetative micronucleus, but also in the exconjugant micronucleus at the same time that rampant excision and breakage are occurring in the adjacent developing macronucleus. Because of this inertness of the micronucleus, we suggest that the first 
steps of transposition do not occur there but, instead, occur in the developing macronucleus and that transposition only finishes in the micronucleus. Thus, we envision a conservative transposition pathway in which the first intermediate is produced by transposase in massive quantities. Completion of transposition, which presumably is relatively infrequent, occurs only if an excised TBEl persists long enough to be translocated into the micronucleus and integrated. We were first led to the idea of movement of DNAs from the macronucleus to the micronucleus when we observed micronuclear regions that most readily were explained as the result of rare accidental insertions of intact macronuclear chromosomes in the micronuclear genome (Dawson and Herrick 1984).

The presence of $\mathrm{dC}_{4} \mathrm{~A}_{4}$ repeats at the tips of the inverted terminal repeats of TBEls strongly implies that a transposition intermediate existed as a free linear DNA with telomeres at least once in evolution (see Herrick et al. 1985). Because the telomeric sequences are strongly conserved throughout the TBE1 family, we believe that a telomere-bearing linear is a routine transposition intermediate. It may be that the telomeric repeats protect TBEls from elimination or that they facilitate limited TBE1 replication. In either case, TBE1s could persist for a longer time, thereby increasing the chances of transport to the micronucleus.

TBE1 homologous sequences have been detected in Oxytricha trifallax (R.L. Hammersmith and G. Herrick, unpubl.), and exconjugant DNA has been examined for free TBE1 forms without success (G. Herrick, unpubl.). Very recently, free forms of both a short IES and Tecl elements have been detected in developing $E$. crassus micronuclei (Jahn et al. 1989; Tausta and Klobutcher 1989). Given the E. crassus results, we are eager to look more thoroughly for free linear TBEls in O. trifallax exconjugants.

In summary, two TBEls and possibly many more are precisely excised from the genome during macronuclear development. This excision process possibly is mediated by transposon genes, may be responsible for all IES excisions, and also may represent the first step of transposition.

\section{Materials and methods \\ Gel electrophoresis analyses}

Electrophoresis and Southern blot transfers were as described by Cartinhour and Herrick (1984), except that blots presented in Figures 2-4 were onto GeneScreen membranes (DuPont) and UV cross-linked using a Stratolinker lamp (Stratagene). Hybridization with restriction fragment probes was as described (Cartinhour and Herrick 1984) for Figure 6, or hybridized by the method of Church and Gilbert (1984) and washed in SSC solutions, as described previously (Cartinhour and Herrick 1984) for Figure 3. Oligonucleotide-probed blots were hybridized and rinsed by the method of Church and Gilbert (1984). DNAs were isolated from gels, either as described earlier (Cartinhour and Herrick 1984) or by using Geneclean glass milk, according to the instructions of the supplier (Bio 101).

\section{Hybridization probes}

Restriction fragments were ${ }^{32} \mathrm{P}-$ labeled either by nick-translation (Maniatis et. al. 1975) or by the random primer reaction (Feinberg and Vogelstein 1983, 1984). Oligomers were 5'-32P-labeled as described previously (Herrick et al. 1987a).

\section{Oligomers}

Oligomers were synthesized with an Applied Biosystems instrument. Oligomers with sequences that occur identically in all three versions: PCR-L1, 5'-dGAAGAATGTGTCGAAACTATAC; PCR-R1, 5'-dCTTAATTGAAATAATTTGTAATAAAC; PE-B，5'-dAGATCTGATTCTAACCATATCGAGTGG; PE-C, $5^{\prime}$-dGCATGAGTTATGAGAGCAAT (AAT is the triplet duplicated by TBE1-2 insertion); T1, 5 -dCTGCATAGAATGATCCAAAGT (AAT is the triplet duplicated by TBE1-1 insertion). vC-specific oligomers: oCl-88, 5'-dTGTTGAGAGAGAACTTTATATAAG (the homologous $v B$ and vA sequences are $5^{\prime}$-dGGTTGAGAGAGCTTTTTGGATAAC and 5'-dAATTGAGAGATGTTTTTGGATAAC, respectively; underlined nucleotides are unique); oC-RCR, 5'-dGAAATTTTAGAAATCA / the homologous $\mathrm{vB}$ and vA sequences are $5^{\prime}$ dGEAATTTTATAAATCG and 5'-dTAAATTTTATAAATCG, respectively). ITR-19: 5'-dCCCAACCCCTAACAAATTC (Herrick et al. 1985).

\section{O. fallax DNAs}

Micronuclear and macronuclear DNAs were isolated, as described previously (Dawson and Herrick 1982), from starved, sterile cultures of the subkaryonide 3.5 (Cartinhour and Herrick 1984). Whole-cell DNAs were isolated from starved open cultures of the young karyonides 9D1 and 9D2, washed, and lysed as if they were macronuclei. The three lines, 3.5, 9D1, and 9D2 represent independent macronuclear reorganization events (Cartinhour and Herrick 1984).

\section{$P C R$ and sequencing of $P C R$ products}

PCR reactions (Saiki et al. 1985) were conducted according to the protocols supplied with the AmpliTaq DNA polymerase (Perkin-Elmer Cetus) but with $50 \mu \mathrm{l}$ reactions, using a PerkinElmer Cetus Thermal Cycler. Production of vC-specific macronuclear DNA involved three rounds of PCR, each followed by $\mathrm{ClaI}$ digestion and gel isolation of the ClaI-resistant material. Primers $(1 \mu \mathrm{M})$ were PCR-LI and PCR-RI. One round of PCR consisted of $10 \mathrm{~min}$ at $94^{\circ} \mathrm{C}$, followed by 30 cycles of denaturation ( $1 \mathrm{~min}$ at $94^{\circ} \mathrm{C}$ ), annealing $\left(1 \mathrm{~min}\right.$ at $\left.37^{\circ} \mathrm{C}\right)$, and polymerization $\left(3 \mathrm{~min}\right.$ at $\left.72^{\circ} \mathrm{C}\right)$, and finished by another $7 \mathrm{~min}$ at $72^{\circ} \mathrm{C}$. The template in the first round of PCR was $50 \mathrm{ng}$ of 3.5 macronuclear DNA. The entire reaction was adjusted to $10 \mathrm{mM}$ $\mathrm{MgCl}_{2}$, cut with ClaI, and subjected to electrophoresis through a $2.0 \%$ agarose gel. Approximately one-third of the DNA was present in the uncut $\sim 570$-bp band. This band was cut from the gel and isolated using Geneclean glass milk (Bio 101). A portion $(\sim 10 \mathrm{ng}$ ) was subjected to a second round of PCR, and all the ClaI-resistant material was again gel isolated. This PCR-ClaI procedure was applied a third time, and the sequence was determined as described in Thein (1989). Dideoxy-termination DNA sequencing was conducted using the Sequenase kit, version 2.0 (United States Biochemical) and $\left[\alpha^{-35}\right]$ dATP (1000 Ci/ mmole; Amersham). Products were analyzed by autoradiography of $6 \%$ polyacrylamide gels, as described (Herrick et al. 1985). Short regions left of the BgIII site have been sequenced for cloned macronuclear $\mathrm{vB}$ and micronuclear vC DNAs using the same techniques. GenBank (Los Alamos National Labora- 
tory) accession numbers for updated files containing those sequences are Ml3031 (vB) and M13037 (vC). The vC macronuclear DNA sequence GenBank file has accession number M25391.

\section{Note added in proof}

Recent PCR results with DNA from the young karyonide 9D1 eliminate the possibility that a TBE1-2-less VC micronuclear DNA exists, which could give rise to vC macronuclear DNA without the need to excise TBE1-2. That is, $\mathrm{vC}$ macronuclear DNA must have been generated from a TBE1-2-bearing precursor, indicating that TBE1-2 is an IES.

\section{Acknowledgments}

We thank Larry Klobutcher and Carolyn Jahn for communication of results prior to publication. We thank many people for valuable discussions and suggestions over the years and for critiques of the manuscript, including Dean Dawson, Larry Klobutcher, Dana Carroll, Gordon Lark, and Jon Seger. The work was supported by grant GM-25203 from the National Institutes of Health.

\section{References}

Ammermann, D. 1988. DNA damage and repair in Stylonychia lemnae (ciliata, protozoa). I. Protozool. 35: 264-267.

Austerberry, C.F. and M.-C. Yao. 1987. Nucleotide sequence structure and consistency of a developmentally regulated DNA deletion in Tetrahymena thermophila. Mol. Cell. Biol. 7: 435-443.

Austerberry, C.F., R.O. Snyder, and M.-C. Yao. 1989. Sequence microheterogeneity is generated at junctions of programmed DNA deletions in Tetrahymena thermophila. Nucleic Acids Res., (in press).

Baird, S.E., and L.A. Klobutcher. 1988. Genetic characterization and use of a restriction fragment length variant in the hypotrichous ciliate Euplotes crassus. J. Protozool. 35: 459-465.

Baird, S.E., G.M. Fino, S.L. Tausta, and L.A. Klobutcher. 1989. Micronuclear genome organization in Euplotes crassus: A transposonlike element is removed during macronuclear development. Mol. Cell. Biol. 9: 3793-3807.

Brunk, C.F. 1986. Genomic reorganization in Tetrahymena. Int. Rev. Cytol. 99: 49-83.

Bruns, P.J. 1986. Genetic organization of Tetrahymena. In The molecular biology of ciliated protozoa (ed. J.G. Gall), pp. 27-44. Academic Press, Orlando, Florida.

Cartinhour, S.W., and G.A. Herrick. 1984. Three different macronuclear DNAs in Oxytricha fallax share a common sequence block. Mol. Cell. Biol. 4: 931-938.

Cherry, J.M., and E.H. Blackburn. 1985. The internally located telomeric sequences in the germ line chromosomes of $\mathrm{Te}$ trahymena are at the ends of transposon-like elements. Cell 43: 747-758.

Church, G.M., and W. Gilbert. 1984. Genomic sequencing. Proc. Natl. Acad. Sci. 81: 1991-1995.

Coen, E.S., T.P. Robbins, J. Almeida, A. Hudson, and R. Carpenter. 1989. Consequences and mechanisms of transposition in Antirrhinum majus. In Mobile DNA led. D.E. Berg and M.M. Howe), pp. 413-436. American Society for Microbiology, Washington, D.C..

Dawson, D. and G. Herrick. 1982. Micronuclear DNA sequences of Oxytricha fallax homologous to the macronuclear inverted terminal repeat. Nucleic Acids Res. 10: $2911-2924$.
1984. Rare internal $\mathrm{dC}_{4} \mathrm{~A}_{4}$ repeats in the micronuclear genome of Oxytricha fallax. Mol. Cell. Biol. 4: 2661-2667.

Dente, L., G. Cesareni, and R. Cortese. 1983. pEMBL: A new family of single stranded plasmids. Nucleic Acids Res. 11: $1645-1655$.

Engels, W.R. 1989. P elements in Drosophila melanogaster. In Mobile DNA (ed. D.E. Berg and M.M. Howe), pp. 437-484. American Society for Microbiology, Washington, D.C.

Fedoroff, N.V. 1989. Maize transposable elements. In Mobile DNA (ed. D.E. Berg and M.M. Howe), pp. 375-411. American Society for Microbiology, Washington, D.C.

Feinberg, A.P., and B. Vogelstein. 1983. A technique for radiolabeling DNA restriction endonuclease fragments to high specific activity. Anal. Biochem. 132: 6-13.

1984. A technique for radio-labeling DNA restriction endonuclease fragments to high specific activity laddendum). Anal. Biochem. 137: 266-267.

Gall, J.G. (ed.) 1987. The molecular biology of ciliated protozoa. Academic Press, Orlando, Florida.

Herrick, G., S. Cartinhour, D. Dawson, D. Ang, R. Sheets, A. Lee, and K. Williams. 1985. Mobile elements bounded by $\mathrm{C}_{4} \mathrm{~A}_{4}$ telomeric repeats in Oxytricha fallax. Cell 43: 759768.

Herrick, G, D. Hunter, K. Williams, and K. Kotter. 1987a. Alternative processing during development of a macronuclear chromosome family in Oxytricha fallax. Genes Dev. 1: $1047-1058$.

Herrick, G., S.W. Cartinhour, K.R. Williams, and K.P. Kotter. 1987b. Multiple sequence versions of the Oxytricha fallax 81-MAC alternate processing family. I. Protozool. 34: 429434.

Jahn, C.L., L.A. Nilles, and M.F. Krikau. 1988. Organization of the Euplotes crassus micronuclear genome. I. Protozool. 35: 590-601.

Jahn, C.L., M.F. Krikau, and S. Shyman. 1989. Developmentally coordinated en masse excision of a highly repetitive element in Euplotes crassus. Cell, (in press).

Kleckner, N. 1989. Transposon Tn10. In Mobile DNA led. D.E. Berg and M.M. Howe), pp. 227-268. American Society for Microbiology, Washington, D.C.

Klobutcher, L.A., C.L. Jahn, and D.M. Prescott. 1984. Internal sequences are eliminated from genes during macronuclear development in the ciliated protozoan Oxytricha nova. Cell 36: $1045-1055$.

Lambowitz, A.M. 1989. Infectious introns. Cell 56: 323-326.

Løvlie, A., B.L. Haller, and E. Orias. 1988. Molecular evidence for somatic recombination in the ribosomal DNA of Tetrahymena thermophila. Proc. Nat1. Acad. Sci. 85: 5156-5160.

Maniatis, T., A. Jeffrey, and D.G. Kleid. 1975. Nucleotide sequence of the rightward operator of phage lambda. Proc Natl. Acad. Sci. 72: 1184-1188.

Moerman, D.G., and R.H. Waterston. 1989. Mobile elements in Caenorhabditis elegans and other nematodes. In Mobile DNA (ed. D.E. Berg and M.M. Howe), pp 537-556. American Society for Microbiology, Washington, D.C.

Ribas-Aparicio, R.M., J.J. Sparkowski, A.E. Proulx, J.D. Mitchell, and L.A. Klobutcher. 1987. Nucleic acid splicing events occur frequently during macronuclear development in the protozoan Oxytricha nova and involve the elimination of unique DNA. Genes Dev. 1: 323-336.

Saiki, R.K., S.J. Scharf, F. Faloona, K.B. Mullis, G.T. Horn, H.A. Erlich, and N. Arnheim. 1985. Enzymatic amplification of $\beta$-globin genomic sequences and restriction site analysis for diagnosis of sickle cell anemia. Science 230: 1350-1354.

Scazzocchio, C. 1989. Group I introns: Do they only go home? Trends Genet. 5: 168-172. 
Steinbrück, G. 1986. Molecular reorganization during nuclear differentiation. In Results and problems in cell differentiation (ed. W. Hennig), vol. 13, pp. 105-174. Springer-Verlag, Berlin.

Sweeney, R., and M.-C. Yao. 1989. Identifying functional regions of rRNA by insertion mutagenesis and complete gene replacement in Tetrahymena thermophila. EMBO $\mathrm{J}$. 8: $933-938$.

Tausta, S.L. and L.A. Klobutcher. 1989. Detection of circular forms of eliminated DNA during macronuclear development in Euplotes crassus. Cell (in press).

Yaeger, P.C., E. Orias, W.-L. Shaiu, D.D. Larson, and E.H. Blackburn. 1989. The replication advantage of a free linear rRNA gene is restored by somatic recombination in Tetrahymena thermophila. Mol. Cell. Biol. 9: 452-460.

Yao, M.-C. 1989. Site-specific chromosome breaking and DNA deletion in ciliates. In Mobile DNA led. D.E. Berg and M.M. Howel, pp. 715-734. American Society for Microbiology, Washington, D.C.

Yu, G.-L., M. Hasson, and E.H. Blackburn. 1988. Circular ribosomal DNA plasmids transform Tetrahymena thermophila by homologous recombination with endogenous macronuclear ribosomal DNA. Proc. Natl. Acad. Sci. 85: 5151-5155. 


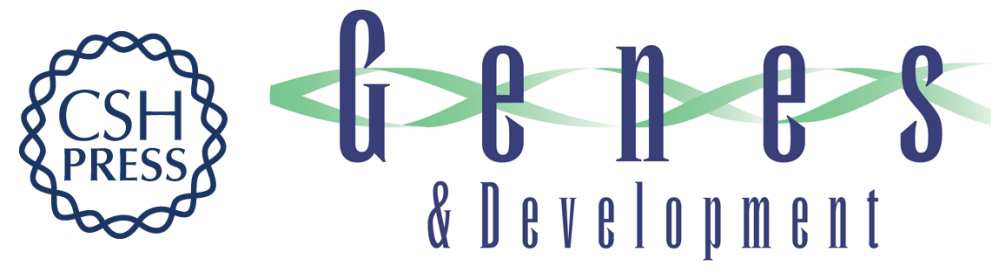

\section{Precise excision of telomere-bearing transposons during Oxytricha fallax macronuclear development.}

D J Hunter, K Williams, S Cartinhour, et al.

Genes Dev. 1989, 3:

Access the most recent version at doi:10.1101/gad.3.12b.2101

References This article cites 27 articles, 12 of which can be accessed free at: http://genesdev.cshlp.org/content/3/12b/2101.full.html\#ref-list-1

License

Email Alerting

Service

Receive free email alerts when new articles cite this article - sign up in the box at the top right corner of the article or click here.

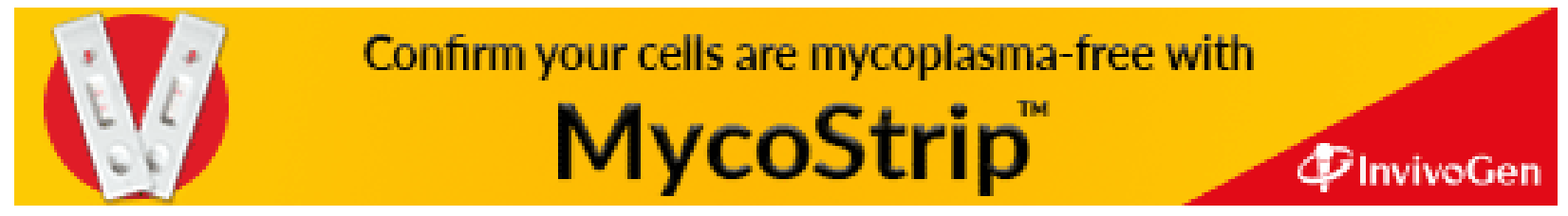

